\title{
The Ponzo illusion and the perception of orientation
}

\author{
WILLIAM PRINZMETAL, ARTHUR P. SHIMAMURA, and MICHELLE MIKOLINSKI \\ University of California, Berkeley, California
}

\begin{abstract}
A new theory, called the tilt constancy theory, claims that the Ponzo illusion is caused by the misperception of orientation induced by local visual cues. The theory relates the Ponzo illusion-along with the Zöllner, Poggendorff, Wündt-Hering, and cafe wall illusions- to the mechanisms that enable us to perceive stable orientations despite changes in retinal orientation or body orientation. In Experiment 1, the magnitude of the misperception of orientation was compared with the magnitude of the Ponzo illusion. In Experiment 2, predictions of the tilt constancy theory were compared with accounts based on (1) low spatial frequencies in the image, (2) memory comparisons (pool-and-store model), and (3) relative size judgments. In Experiment 3, predictions of the tilt constancy theory were tested against predictions of the assimilation theory of Pressey and his colleagues. In the final experiment, the orientation account was compared with theories based on linear perspective and inappropriate size constancy. The results support the tilt constancy theory.
\end{abstract}

Illusions have long been considered to provide insights into normal visual processing. Thus Richard Gregory asked, "Can it be that illusions arise from informationprocessing mechanisms that under normal circumstances make the visible world easier to comprehend?" (Gregory, 1968, p. 66). A critical issue has been which normal information-processing mechanisms are reflected by specific illusions. The Ponzo illusion, illustrated in Figure 1 , has been claimed to reflect many different fundamental visual processes, from spatial frequency filtering in cortical area V1 to the linear perspective and the inappropriate application of size constancy.

We propose a new theory of the Ponzo illusion that is based on the mechanisms of orientation perception. Specifically, we propose that the Ponzo illusion is due to the misperception of orientation induced by local visual cues. A consequence of the misperception of orientation is that the bar near the apex (i.e., on the right) of the Ponzo illusion illustrated in Figure 1 appears longer than the bar near the base (i.e., the bar on the left). We will suggest that the mechanisms that normally help us perceive constant orientation despite changes in retinal orientation (tilt constancy) may underlie this illusion. In this report, we will first present this new account of the Ponzo illusion. Second, we will test our theory against theories based on linear perspective and size constancy (e.g., Gillam, 1980; Gregory, 1963), low-pass filtering (Ginsburg, 1984), assimilation (Girgus \& Coren, 1982; Pressey \& Epp, 1992), and relative size comparisons (Künnapas,

This research was supported by NIH Grants MH48757 and NS17778 to A.P.S. and NSF Grant SBR-9319103 to W.P. We thank Edward Hubbard, Diane Beck, Susan Ravizza, Rolf Nelson, Guha Jayachandran, and Abigail Bautista for their helpful comments on the manuscript. Correspondence concerning this article should be sent to W. Prinzmetal, Department of Psychology, University of California, Berkeley, CA 947201650 (e-mail: wprinz@socrates.berkeley.edu).
1955). Finally, we will extend our theory to account for other illusions, some of which are illustrated in Figure 1.

The role of local visual cues in the perception of orientation was illustrated in a classic study by Gibson (1937). This effect is termed the tilt induction effect. Observers were presented a stimulus such as that in Figure $2 \mathrm{~A}$. The task was to set the shorter line to be vertical. Gibson found that the observers distorted their adjustments in the direction of the context lines. That is, the tilted context distorted the observer's sense of vertical. Thus, with stimuli such as that in Figure 2A, the observers perceived the vertical line as tilted in a counterclockwise direction and therefore adjusted it too far in a clockwise direction to make it appear vertical. The opposite was true of stimuli like that shown in Figure $2 \mathrm{~B}$. When the context is presented before the line to adjust, the effect is called the tilt-after effect (Gibson \& Radner, 1937).

The Zöllner illusion has long been considered an example of the tilt induction effect (Day, 1972; Howard, 1982, pp. 156-157). In Figure 1 (Zöllner illusion), the horizontal lines appear slanted in the direction opposite the context lines. Note that the effect of context is local, so that in Figure 1, the context lines on the top determine the perceived orientation of the line on the top and those on the bottom determine the perceived orientation of the line on the bottom.

We propose that the Ponzo illusion is caused by the same mechanism as the tilt induction effect and the Zöllner illusion. To understand the relation between the tilt induction effect and the Ponzo illusion consider Figure 3. In Figure 3A, because of the tilt induction effect, the vertical line will appear slanted in the direction opposite the slanted line (i.e., it will appear tilted in a counterclockwise direction). We have shown that this effect will cause the dot on the top to be perceived as shifted to the left, as compared with the dot on the bottom (Shimamura \& Prinzmetal, 2000). In Figure 3B, the dot on the top will appear 


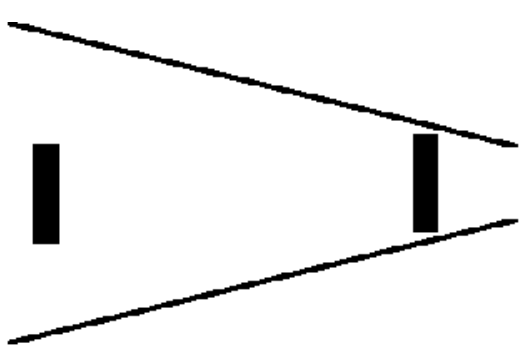

Ponzo Illusion

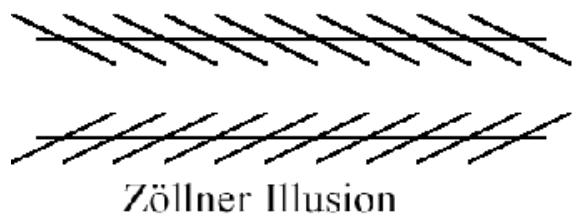

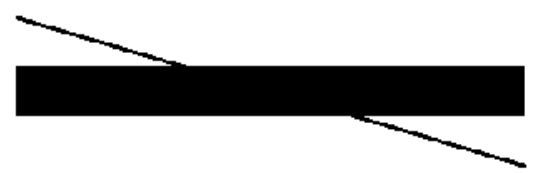

Poggendorff Illusion

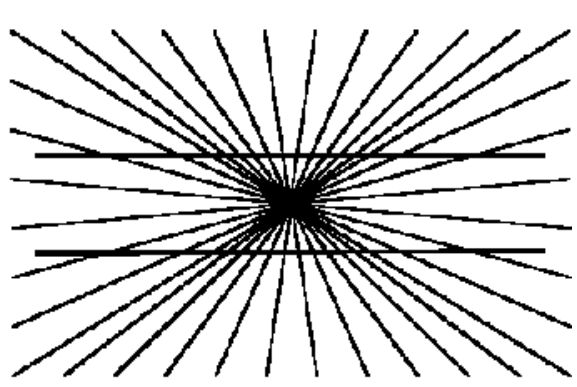

Wündt-Hering Illusion

Figure 1. A variety of visual illusions that can be accounted for by the misperception of orientation.

shifted to the right, as compared with the bottom dot. Figure 3C simply puts the two illusions together and connects the dots in a different manner. The consequence of the misperception of the location of the dots is that the top horizontal line will appear longer than the bottom horizontal line. In Experiment 1, we test whether the misperception of orientation caused by the tilt induction effect (illustrated in Figures 3A and 3B) is of sufficient magnitude to account for the Ponzo illusion (Figure 3C).

Figure 4 illustrates the relation between the Ponzo and the Zöllner illusions. In the Zöllner illusion, the distance between the long lines appears larger on the right, whereas in the Ponzo illusion the vertical line on the right appears longer. The lower panel superimposes the two illusions and illustrates that the Ponzo illusion is implicit in the

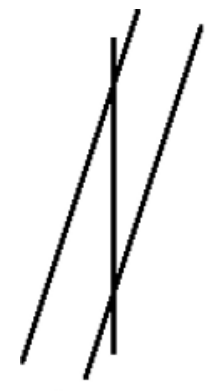

A

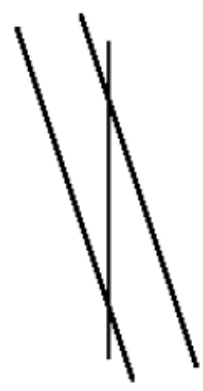

B
Figure 2. The tilt induction effect. (A) The vertical line appears slightly slanted in a counterclockwise direction. (B) The vertical line appears slightly slanted in a clockwise direction. The figure is drawn to suggest a relation between the tilt induction effect and the Zöllner illusion.
Zöllner illusion. In the Zöllner illusion, the oblique context lines cause the right end of the horizontal lines to appear farther apart than the left end. In the Ponzo illusion, the oblique lines cause the ends of the vertical line on the right to appear farther apart than the ends of the vertical line on the left. The consequence of this distortion in the Ponzo illusion is that the vertical line on the right appears longer than the one on the left (see Figure 1). Described in this manner, the relation between the Zöllner and the Ponzo illusions could be considered a hidden-figures task: One can see little Ponzo figures hidden in the Zöllner illusion.

There are a number of similarities between the Ponzo illusion, on the one hand, and the Zöllner and tilt induction illusions, on the other. For example, Gibson (1937) showed that the tilt induction effect was strongest when the contest line was from $15^{\circ}$ to $20^{\circ}$ from vertical or horizontal (see also Andriessen \& Bouma, 1976; Kramer, 1978; Shimamura \& Prinzmetal, 2000). Similarly, the Zöllner illusion is greatest when the angle between the test and the context lines is in this same range (e.g., Oyama, 1975; Wallace \& Moulden, 1973). Note that the Ponzo illusion is greatest when each of the oblique lines is $15^{\circ}-20^{\circ}$ from vertical or horizontal (Fisher, 1968b, 1973). Gibson noted that the tilt induction effect is greater when the context lines are near vertical than when they were near horizontal. Similarly, both the Zöllner and the Ponzo illusions are greater in the vertical than in the horizontal orientation (Fisher, 1968b, 1973; Oyama, 1975). We do not know the cause of this difference, but it may be related to other anisotrophies in the visual field (Prinzmetal \& Gettleman, 1993). Of course, these similarities may simply be a coincidence, and the misperception of 


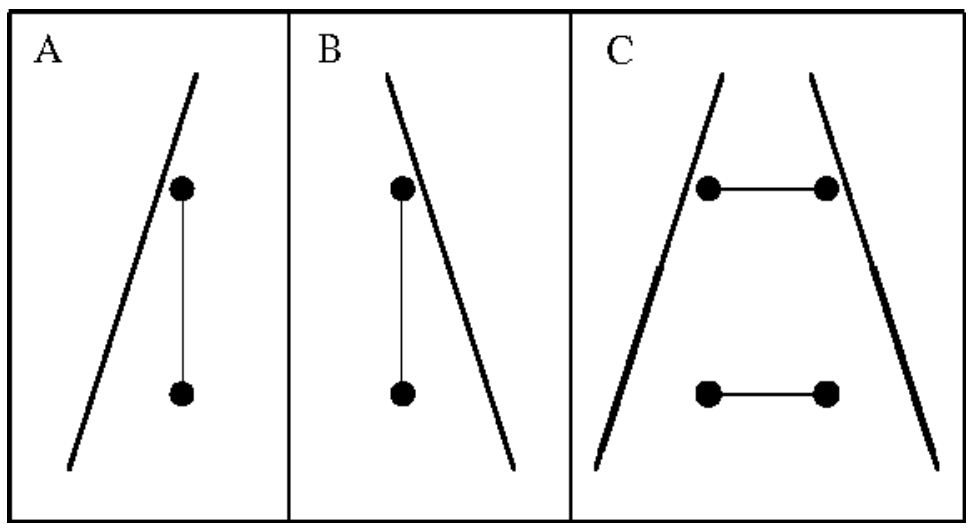

Figure 3. The relation between the tilt induction effect and the Ponzo illusion is illustrated. In panel $A$, the tilt induction effect causes the top dot to appear slightly to the left of the bottom dot. In panel $B$, the top dot appears slightly to the right of the bottom dot. Panel $\mathrm{C}$ combines these effects to create the Ponzo illusion.

length in the Ponzo illusion might be unrelated to orientation perception. The goal of this paper is to directly test this account of the Ponzo illusion.

Our account of the Ponzo illusion is part of a more general theory that we call the tilt constancy theory (see Prinzmetal \& Beck, in press; Shimamura \& Prinzmetal, 2000). We will present the theory in more detail in the General Discussion section. Briefly, by tilt constancy, we mean the ability to perceive vertical and horizontal despite changes in retinal orientation. We have also called this approach the orientation framing theory (Shimamura \& Prinzmetal, 2000), but here we use the term tilt constancy to emphasize the adaptive significance of the mechanisms that underlie these illusions. In the tilt induction effect, visual cues (the oblique lines) cause errors in the perception of orientation, but generally visual cues to orientation provide veridical and stable information about vertical and horizontal, despite changes in retinal orientation. Although the goal of the present paper is to test the tilt constancy theory of the Ponzo illusion, we will suggest in the General Discussion section that the theory can also account for a number of other illusions.

In Experiment 1, we compared the magnitude of the misperception of orientation with the magnitude of the Ponzo illusion, using the same observers and almost identical stimuli. This experiment illustrates that the misperception of orientation is of the appropriate magnitude to account for the Ponzo illusion.

In Experiments 2, 3, and 4, we compared our tilt constancy theory of the Ponzo illusion with previous theories of this illusion. The theories that we considered in this paper are (1) the low-pass filter theory (Ginsburg, 1984), (2) the assimilation theory (Pressey \& Epp, 1992), (3) a size-comparison theory based on the work of Kün-

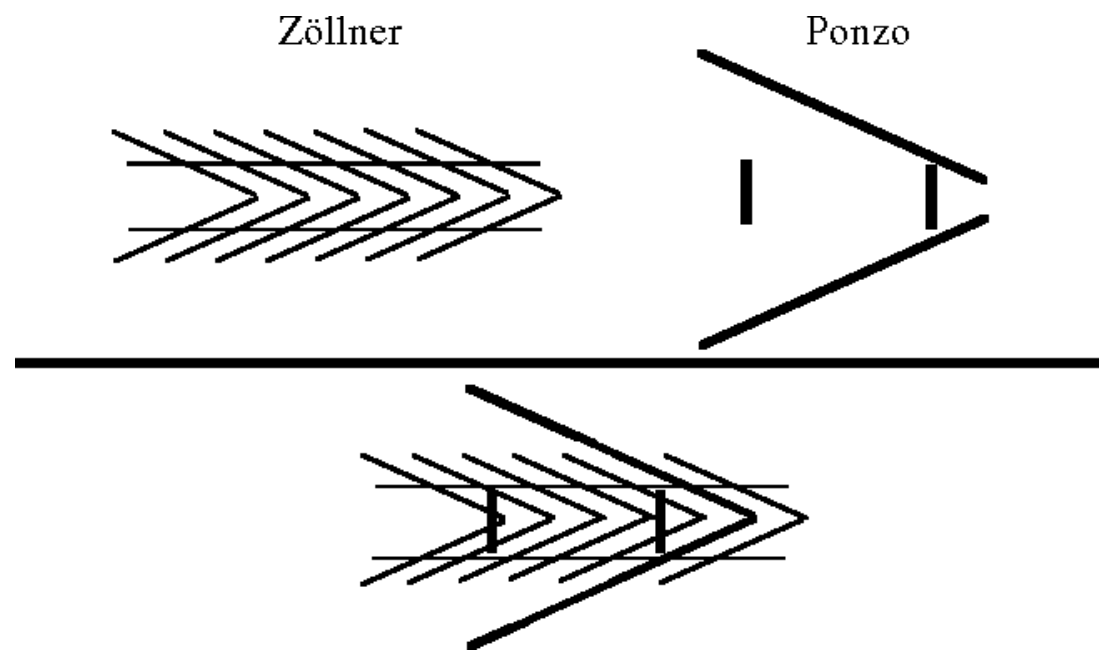

Figure 4. The relation between the Zöllner and the Ponzo illusions. The top panel illustrates the two illusions separately. In the bottom panel, the illusions are superimposed. Note that the direction of the misperception of space is the same in both illusions. 
napas and others (Künnapas, 1955), (4) the pool-andstore theory of Girgus and Coren (1982), and (5) a family of theories that make reference to linear perspective and size constancy (e.g., Gillam, 1980; Gregory, 1963). Although this list of theories is not complete, they may be the most prominent theories of the Ponzo illusion, and they are reasonably diverse. The present experiments were designed to contrast predictions of each of these theories with predictions based on the misperception of orientation. Each of these theories will be discussed in more detail within the context of the experiments that follow.

\section{EXPERIMENT 1}

In Figure 5A, the top horizontal line appears longer than the bottom horizontal line. We propose that this is because, in making length judgments, observers, to some extent, are making judgments about the orientation of two virtual lines connecting the ends of the horizontal lines. If these virtual lines appeared vertical, the horizontal lines would appear to be the same length. However, these virtual lines do not appear vertical, and therefore the horizontal lines do not appear to be the same length. The sense of vertical orientation of the virtual lines is affected by the nearest oblique line.

The issue addressed in this experiment is whether the misperception of vertical alignment is of sufficient magnitude to account for the Ponzo illusion. To measure the Ponzo illusion, we presented observers with stimuli like that in Figure 5A. The observers' task was to adjust the length of the bottom horizontal line to match the length of the top horizontal line. (As the observers adjusted the line, it changed at both ends.) Most of the observers overestimated the length of the top line and thus made the length of the line on the bottom too long.

In designing a task to measure observers' sense of alignment, we had two criteria. First, we wanted the stimuli to be similar to the Ponzo stimuli (Figure 5A). Second, we wanted to make the task so that it was impossible for observers to do the task by matching line length. These criteria were met with the stimulus illustrated in Figure 5B. The observers adjusted the length of the line on the bottom so that the free end of the line was vertically aligned with the end of the top horizontal line.

In summary, the claim is that when making length judgments in Figure 5A, observers are attempting to align the end of the lines and the sense of alignment is affected by the oblique context lines. Hence, the magnitude of the error from the alignment task (Figure 5B) should predict the magnitude of the Ponzo illusion.

\section{Method}

Procedure. The method of adjustment was used for both the Ponzo illusion task and the alignment task. To measure the Ponzo illusion (Figure 5A), on each trial, the observers adjusted the length of the bottom horizontal line to match the length of the top horizontal line, using the up and down arrow keys on a computer keyboard. Each time the observer pressed the up arrow key, the base line got longer by 1 pixel but was centered between the context lines. ${ }^{1}$ In a similar manner, the down arrow key made the bottom line shorter. The observers were instructed to take their time. When they were satisfied with their adjustment, they pressed the return key. Each subject was tested for 24 trials.

For the alignment task, the observers were presented with stimuli similar to that in Figure 5B. The task was to adjust the free end of the bottom line so that it was "vertically aligned, according to gravity" with the free end of the top line. On half of the trials, the free end was on the right side of the figure (as in Figure 5B), and on half the trials, the free end was on the left side of the figure. Each subject was tested for a total of 24 trials, and the order of the leftand right-hand versions was randomly determined.

The initial setting of the bottom line was randomly chosen from a rectangular distribution of lengths from $50 \%$ shorter than the top horizontal line to $50 \%$ longer than this line. Each observer participated in both tasks, with half the observers beginning with the Ponzo task. The experiment took about $10 \mathrm{~min}$.

Stimuli. The stimuli were presented on a 17-in. Apple Macintosh monitor set at $832 \times 624$ pixels. The stimuli were drawn in black on a white background. The observers sat $40 \mathrm{~cm}$ from the monitor,

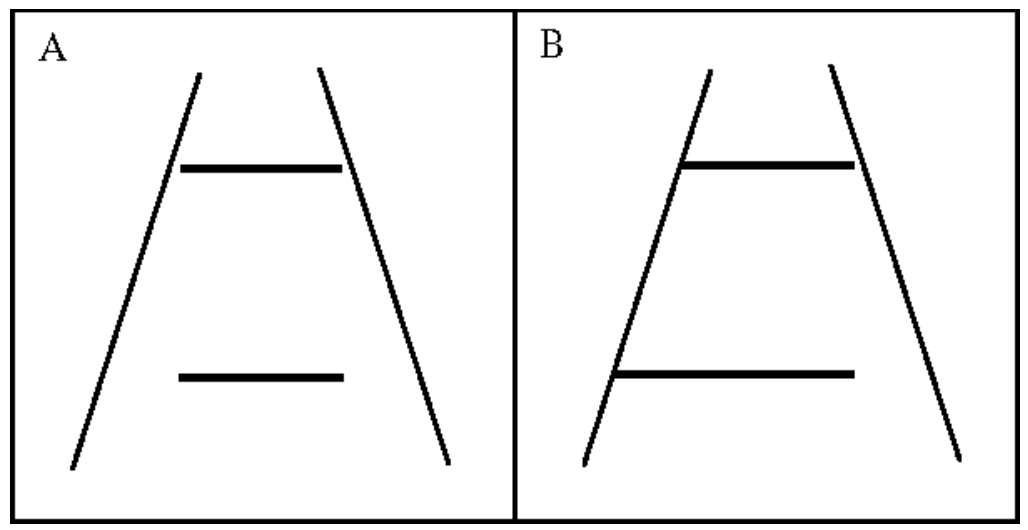

Figure 5. The stimuli used in Experiment 1. (A) In the Ponzo task, observers adjusted the length of the bottom horizontal line to match the length of the top line. (B) In the alignment task, observers adjusted the length of the bottom line so that its end was vertically aligned with the end of the top line. In this way, they indicated what they perceived as vertical. The stimuli are drawn to scale. 
with their head restrained with a chinrest. The stimuli in Figure 5 are drawn to scale. The horizontal lines were 4 pixels wide. In the Ponzo task, the top (standard) line was 120 pixels long $(4.2 \mathrm{~cm})$, subtending a visual angle of $6.1^{\circ}$. The initial setting of the bottom line was randomly determined and was from 60 to 180 pixels (from $3.1^{\circ}$ to $9.1^{\circ}$ of visual angle). In the alignment task, the top line was always 128 pixels in length, subtending a visual angle of $6.5^{\circ}$. The initial setting of the length of the bottom line was randomly determined on each trial and was from 64 to 192 pixels in length (from $3.3^{\circ}$ to $9.7^{\circ}$ of visual angle). The oblique context lines were slanted $18.4^{\circ}$ from vertical.

Observers. There were 20 observers recruited from among graduate and undergraduate students in the University of California, Berkeley, psychology department. The observers' ages ranged from 22 to 41 years. Across the experiments reported in this paper, approximately half the observers were male, and half were female.

\section{Results}

In the Ponzo task, the observers adjusted the bottom line too long by an average of 12.8 pixels. This error corresponds to adjusting the line $10.7 \%$ too long, which is typical for the Ponzo illusion. (Note that 20 pixels equals $1^{\circ}$ of visual angle.) Each of the 20 observers exhibited the illusion (e.g., Fisher, 1968b, 1973).

In the alignment task, when the free ends to align were on the left, the observers adjusted the bottom line an average of 5.7 pixels too far to the left. When the free ends were on the right, they adjusted the end of the line an average of 6.0 pixels too far to the right. The difference between the left and the right errors did not approach significance.

If the Ponzo illusion is due to a misperception of alignment, the Ponzo illusion should be similar to the total alignment illusion (left + right misalignment). The magnitude of the Ponzo illusion was indeed close to the total alignment illusion, 12.8 versus 11.7 (i.e., $5.7+6.0$ ) pixels. This difference was not reliable by a paired $t$ test $[t(19)=1.24]$. The standard deviation of the 24 settings, averaged over the 20 observers, was 5.7 and 4.6 pixels for the Ponzo and the alignment tasks, respectively. Hence, the alignment task gives an illusion of a magnitude that almost perfectly predicts the Ponzo illusion. For this group of observers, if we were to predict the magnitude of the Ponzo illusion from performance on the alignment task, we would err by a mere 1.1 pixels $\left(0.056^{\circ}\right.$ of visual angle).

We were worried that the experience of performing one task might change observers' strategy on the other task. For example, having first performed the alignment task, observers might have a different strategy in doing the Ponzo task than they would if they had not performed the alignment task. Hence, we separately analyzed only the first block, which constitutes a between-groups comparison. The difference between the magnitude of the Ponzo illusion and that of the total alignment illusion was not significant $[t(18)=0.83]$. The average error for the Ponzo group was 11.8 pixels, and the average total error for the alignment group was 13.7 pixels.

Finally, if the cause of the Ponzo illusion is a misperception of orientation, observers who evince a large align- ment illusion should also have a large Ponzo illusion. The correlation between tasks was quite high $(r=.71, p<$ .01 ). There could be other reasons for the correlation between tasks, but the high correlation between these two illusions is consistent with the claim that the two illusions are caused by the same mechanism.

\section{Discussion}

This experiment demonstrates that the misalignment owing to the oblique context lines can cause an illusion of the same magnitude as the Ponzo illusion. In a sense, the alignment task is a quantitative model for the Ponzo illusion with no free parameters. We were somewhat surprised at how closely the illusions matched in magnitude. Note that in the alignment task (Figure 5B), it is impossible to use length to perform the tasks, because the bottom line will always be longer. When making the length judgment (i.e., the Ponzo illusion, Figure 5A), observers could use line length and a sense of vertical to make the judgment.

Note that there are two interpretations of our explanation of the Ponzo illusion and the close correspondence between the results of the two tasks. On the one hand, observers may be literally lining up the ends of the lines with what they perceive as vertical. On the other hand, the oblique lines may be distorting the representation of space so that one would obtain a Ponzo illusion even if the ends of the lines did not line up. For example, Post, Welch, and Caufield (1998) found that the Müller-Lyer and Judd illusions are caused by the expansion and/or the contraction of space near the arrowheads. On this interpretation, the oblique lines cause local distortions of space so that the ends of the lines need not be directly in alignment.

Of course, the similarity in magnitude between the illusions could be a coincidence. Furthermore, other theories may also be able to account for the magnitude of the Ponzo illusion (e.g., Pressey, Butchard, \& Scrivner, 1971). Demonstrating that a theory is capable of generating an illusion of the correct magnitude is only weak evidence for that theory. A better approach is to compare directly the predictions of competing theories. In the remaining experiments, we compare predictions of our orientation account of the Ponzo illusion with previous theories of this illusion. Note, however, that if we can demonstrate that other theories do not account for the Ponzo illusions, it is unnecessary to postulate multiple causes of this illusion: The misperception of orientation is of sufficient magnitude to account for the entire Ponzo illusion.

\section{EXPERIMENT 2A}

Experiments 2A and 2B compared the low-pass filter theory (Ginsburg, 1984), the pool-and-store model (Girgus \& Coren, 1982), and a size-comparison theory with the tilt constancy theory. The low-pass filter theory is based on the idea that early in visual processing, the visual system performs operations akin to Fourier analysis (DeValois \& DeValois, 1990), creating a functional sep- 


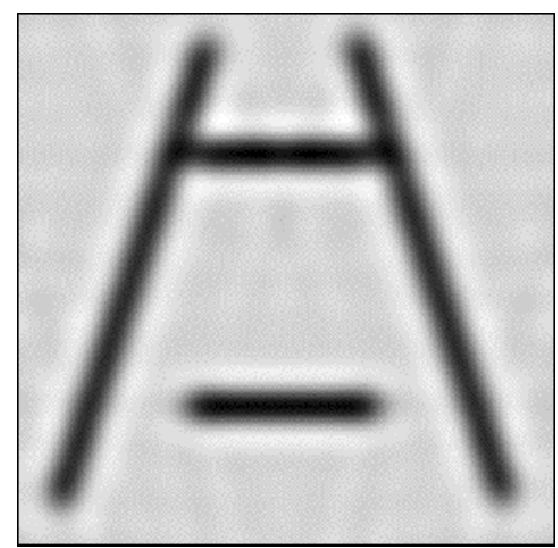

Figure 6. A representation of the low spatial frequencies in the standard Ponzo stimulus. The top horizontal line blurs into and includes part of the context lines, making it longer than the bottom horizontal line.

aration of spatial frequencies. Figure 6 represents some of the low spatial frequencies in the Ponzo figure. In this representation, the top horizontal line blurs into the context lines. Measurements of the length of this line would therefore include part of the context lines. The bottom horizontal line does not blend into the context lines. Hence, the top line would be longer than the base line in the low-frequency representation. The critical stimulus factor is that the gap between the top horizontal line and the context is smaller than the gap between the bottom line and the context. Hence, blurring into the context lines only occurs at the top of the figure. Although the low-pass filter theory has been tested as a cause of the Müller-Lyer and Poggendorff theories (e.g., Carlson, Moeller, \& Anderson, 1984), we do not know of explicit tests of this theory in regard to the Ponzo illusion.

For the pool-and-store model (Girgus \& Coren, 1982), like the low-pass filter theory, the critical stimulus factor is the size of the gap between the parallel lines and the context. At the apex of the figure, where this gap is small, the parallel line and the context are apprehended in a single glance, whereas at the bottom end of the figure, perception of the test line and the context requires more than one glance, according to the pool-andstore model. Objects that are apprehended in a single glance exhibit assimilation (i.e., are pooled), whereas those that are apprehended in different glances require that information be retained (i.e., stored) and result in a contrast effect. The assimilation at the top of the figure is a mechanism similar to Pressey's assimilation model (discussed below), whereas the contrast notion is unique to this theory.

The size-comparison theory is based on the finding that a line in a small rectangle can appear longer than the same line in a large rectangle (e.g., Gogel \& Sturm, 1972; Künnapas, 1955; Rock \& Ebenholtz, 1959). The claim is that observers are making an implicit comparison be- tween the sizes of the gaps at the top and the bottom of the figure. In the Ponzo figure, the gap between the top horizontal line and the context line is smaller than the gap between the bottom horizontal line and the context. Any implicit size comparison would have the consequence of making the top line appear longer.

Hence, the critical stimulus factor for all three theories is the relative size of the gap between the context and the test lines. To test this claim, we compared the standard Ponzo figure (Figure 7A) with what we term the rectilinear version (Figure 7B). The critical factor is that the sizes of the gaps between the parallel lines and the apex line were the same in the two figures. ${ }^{2}$ The length of the base line varied, but the gap size between a given base line and the context was the same in the standard and the rectilinear versions. Because the gap size is the same, all three theories predict that the standard and the rectilinear forms should yield the same size illusion. Note that in several respects, Experiment $2 \mathrm{~A}$ is similar to work by Fisher (1968b, 1973). We will review Fisher's results in the Discussion section.

The tilt constancy theory makes a very different prediction. We propose that the oblique context lines in the Ponzo figure locally affect the perception of vertical and horizontal. The rectilinear version (Figure 7B) does not have any oblique lines. Therefore, we do not predict an illusion in the rectilinear version.

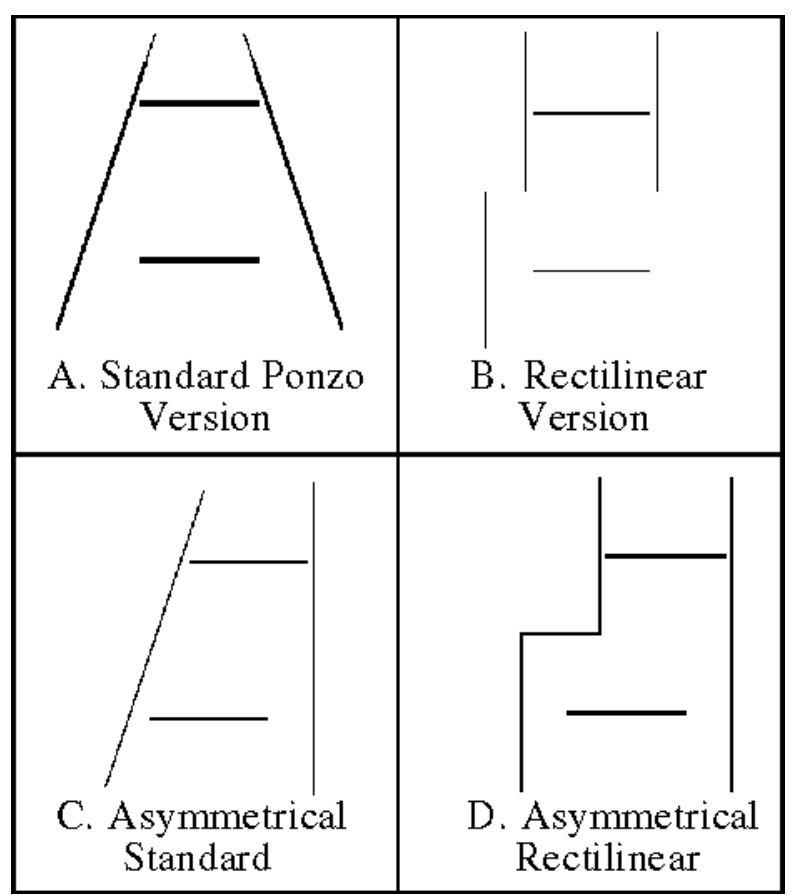

Figure 7. The stimuli used in Experiments 2A and 2B: (A) standard Ponzo figure used in Experiment 2A; (B) rectilinear version used in Experiment 2A; (C) asymmetrical standard Ponzo version used in Experiment 2B; (D) asymmetrical rectilinear stimulus used in Experiment 2B. The stimuli are drawn to scale. 


\section{Method}

Procedure. As in Experiment 1, the method of adjustment was used to measure the Ponzo illusion in the standard version (Figure 7A) or the rectilinear version (Figure 7B). On each trial, the observers were presented with either the standard or the rectilinear version. The top line was the standard, and the task was to adjust the length of the bottom line to match the length of the standard line. The observers adjusted the length of the bottom line as in Experiment 1.

Each observer was tested for 12 trials with the standard Ponzo and for 12 trials with the rectilinear version. The order of trials was randomly determined for each observer. The initial setting of the bottom line was determined as before. The experiment took about $10 \mathrm{~min}$.

Stimuli. The apparatus and stimuli were similar to those used for Experiment 1 . The dimensions of the standard Ponzo were exactly the same as those in Experiment 1. The length of the horizontal lines was the same for the standard and the rectilinear stimuli. The stimuli in Figure 7 are drawn to scale so that overall, the sizes of the standard and the rectilinear Ponzo figures were very similar.

Observers. There were 24 observers recruited from the University of California, Berkeley, psychology department subject pool. They were given course credit for their participation. Each observer participated in several different diverse experiments in a 1-h session, including Experiment 3 (described below). The observers' ages ranged from 18 to 21 years.

\section{Results and Discussion}

Counter to the predictions of the low-pass filter, pooland-store, and size-comparison theories, the standard version of the Ponzo yielded a significantly greater illusion than did the rectilinear version $[9.78 \%$ vs. $0.16 \%$; $t(23)=9.99, p<.01]$. In the standard version, 23 of 24 observers adjusted the bottom line longer than the top line (i.e., a normal Ponzo illusion), whereas with the rectilinear version, 12 observers adjusted the bottom line longer than the standard, while 12 adjusted it shorter. Hence, with the standard version, we obtained a robust illusion, whereas with the rectilinear version, the illusion vanished, as was predicted by the tilt constancy theory.

Before discussing these results, we wanted to test an alternative explanation of the findings. It is possible that there is additional information in the rectilinear version that assists observers in making more veridical settings. Consider Figure 7B. Perhaps observers can mentally project the top vertical lines of the context toward the base lines. This imagined line would provide extra information to help observers make more veridical settings in the rectilinear version than in the standard version. Consistent with this explanation, the observers exhibited less variability in the rectilinear version than in the standard version. The standard deviation of the 12 settings, averaged over the 24 observers, was $4.6 \%$ and $3.6 \%$ illusion for the standard and the rectilinear versions of the Ponzo, respectively. Experiment 2B tested this hypothesis.

\section{EXPERIMENT 2B}

Experiment 2B was similar to Experiment 2A, except that the stimuli were made asymmetrical, as is shown in Figures 7C and 7D. The context in the standard version (Figure 7C) included one oblique line and one vertical line. Fisher (1968a) has demonstrated that a Ponzo illusion can be obtained with a single oblique line of about half the strength of the two-lined version. With a single oblique line and a vertical line, we would also expect an illusion of about half the magnitude as that with the standard version in Experiment 2A, because local orientation perception is only affected on one side of the figure. On the other hand, the low-pass filter, pool-and-store, and the size-comparison theories predict no difference between the standard Ponzo versions in Experiments 2A and 2B (Figures 7A and 7C), because the gaps are approximately the same size.

The critical prediction, however, is between the asymmetrical rectilinear version (Figure 7D) and the asymmetrical standard version (Figure 7C). The low-pass filter, pool-and-store, and size-comparison theories predict the same illusion, because the gaps between the context and the parallel lines are the same for the top horizontal line. Our tilt constancy theory predicts an illusion in the asymmetrical standard version (Figure 7C), but not in the asymmetrical rectilinear version (Figure 7D). Since the parallel lines were offset relative to each other, the criticism discussed above does not apply.

\section{Method}

The procedure was identical to that in Experiment 2A, except that we used asymmetrical versions of the rectilinear and the normal Ponzo illusions (see Figures 7C and 7D). The apex (standard) and base (adjustable) lines were centered between the context lines. Sixteen observers participated.

\section{Results and Discussion}

Consistent with the tilt constancy theory, the asymmetrical standard version of the Ponzo yielded a significantly greater illusion than did the asymmetrical rectilinear version [ $4.67 \%$ vs. $-1.25 \% ; t(15)=4.11, p<.01]$. (The negative number indicates that the observers adjusted the line shorter than the standard.) The standard deviations of the 12 settings, averaged over the 16 observers, were $4.1 \%$ and $7.0 \%$ illusion for the asymmetrical standard and the rectilinear versions, respectively. In the asymmetrical standard version, 14 of 16 observers adjusted the base line longer than the apex line (i.e., a normal Ponzo illusion), whereas with the asymmetrical rectilinear version, 8 observers adjusted the base line longer than the standard, while 8 adjusted it shorter. Comparing the standard version (Experiment 2A, Figure 7A) with the asymmetrical standard version (Experiment 2B, Figure 7C), we obtained an illusion about half the magnitude of that for the standard Ponzo in Experiment 2A $(9.78 \%$ vs. $4.67 \%$ ), but neither rectilinear version yielded an illusion.

Although the tilt constancy theory did not predict an illusion in the rectilinear figures, we have to admit surprise that we did not obtain some illusion with these stimuli. Size framing has been demonstrated many times, and it is a reliable, robust finding (e.g., Gogel \& Sturm, 1972; Künnapas, 1955; Rock \& Ebenholtz, 1959). Thus, one might have expected that whereas both the mechanism of 
orientation and that of size framing would operate in the standard Ponzo figures, size framing would operate in the rectilinear versions. Hence, one might reasonably expect the illusion to be reduced, not eliminated. In fact, Fisher (1968b) found a small framing effect with similar stimuli. Note, however, that Fisher (1973) did not replicate this effect.

Although we do not know why we did not obtain a sizeframing effect, we suggest three possible factors. One possibility is that in comparison with the size-framing experiments cited above, the gap between the parallel lines and the context (particularly the top horizontal line) in our experiment was much smaller than in previous experiments. This factor would suggest a possible dissociation between the Ponzo illusion and size framing. Whereas the Ponzo is greatest when the gap between the apex line and the context lines is small (see, e.g., Fisher, 1969; Jordan \& Randall, 1987), the opposite might be true of size framing. A second possibility is that in the typical size-framing experiment, the large and small frames form different objects, whereas in our figure, they form a single object (cf. Fisher, 1968b). Finally, in Fisher's (1968b) experiment, the context lines were very thick and prominent, as compared with the test lines. Perhaps in that situation, a process like low-pass filtering operates. Finally, note that the low-pass filter theory, the pool-andstore model, and a size-comparison theory all predict no difference in illusion magnitude with the rectilinear and the standard versions of the Ponzo illusion in Experiments $2 \mathrm{~A}$ and $2 \mathrm{~B}$.

We are by no means arguing that size framing does not take place. Nor are we arguing that the visual system does not engage in processes akin to Fourier analysis (De-
Valois \& DeValois, 1990). We are arguing that neither explanation is necessary or sufficient to account for the Ponzo illusion.

\section{EXPERIMENT 3}

Pressey and his colleagues (Pressey et al., 1971; Pressey $\&$ Epp, 1992) proposed that the Ponzo illusion is caused by the same mechanism that causes the Müller-Lyer illusion (see Figure 8A). In the Müller-Lyer illusion, the horizontal shaft connecting the wings out (top of Figure 8A) appears longer than the horizontal shaft connecting the wings in (bottom, Figure 8A). According to Pressey, the Müller-Lyer illusion is caused by a process that assimilates the "wings" of the Müller-Lyer figure with the shaft. Pressey pointed out that the Ponzo figure contains components of the Müller-Lyer figure. These components are shown with solid lines in Figure 8B. To the extent that the components consistent with the Müller-Lyer illusion affect perception in the Ponzo figure, the top horizontal line should appear longer than the bottom line.

The Ponzo figure also contains components that are inconsistent with the Müller-Lyer illusion, however. In Figure 8B, these components are drawn with dashed lines. The line segments inconsistent with the MüllerLyer illusion would tend to work against the Ponzo illusion. The reason that the Müller-Lyer-consistent line segments have a greater effect on perception, according to Pressey, is that while engaged in an experiment, observers are attending to the center of the figure. $\mathrm{Ob}-$ servers' field of attention is illustrated with a gray circle in Figure 8B. Observers process more information in the

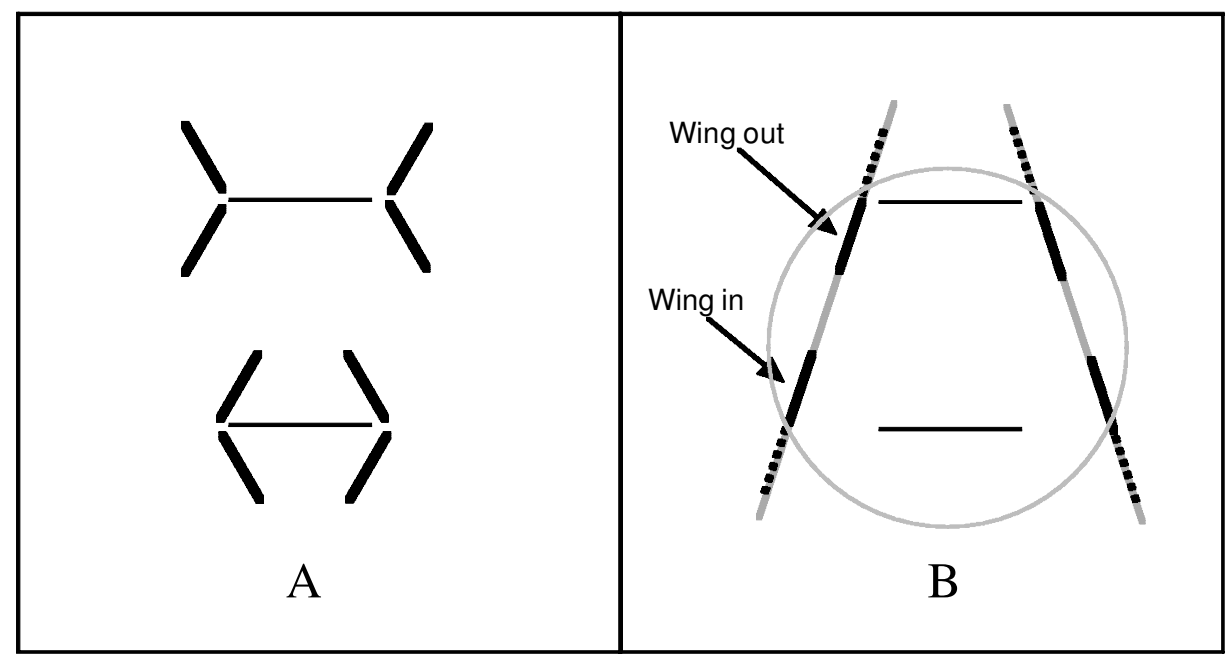

Figure 8. (A) The Müller-Lyer illusion. The shaft connecting the wings out (top) is perceived as longer than the shaft connecting the wings in (bottom). (B) The assimilation theory of the Ponzo illusion. The parts of the figure that are consistent with the Müller-Lyer figure are shown in bold. Observers attend to the center of the figure and thus process more of the Müller-Lyer-consistent information than of the inconsistent information. 


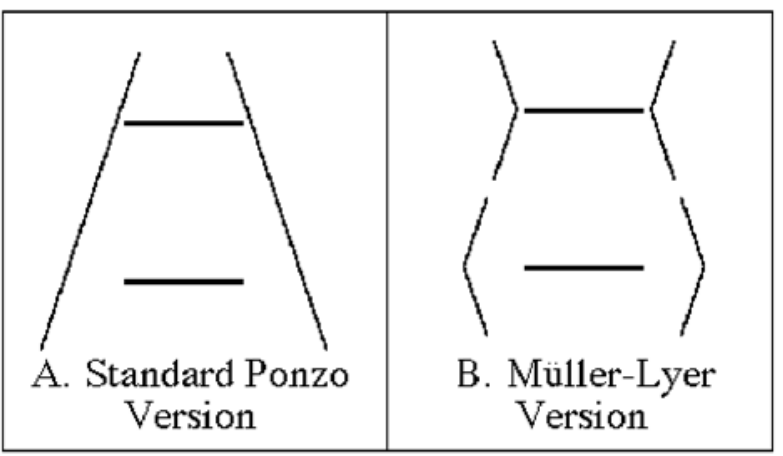

Figure 9. Standard and Müller-Lyer versions of the Ponzo illusion used in Experiment 3.

center of the figure, and hence the Müller-Lyer-consistent line segments have a greater influence than do the inconsistent segments (dashed lines).

We tested the assimilation theory of the Ponzo illusion by comparing the normal Ponzo figure (Figure 9A) with a version that contained only Müller-Lyer consistent line segments (Figure 9B). Regardless of the state of attention, Pressey's theory predicts that the version with only Müller-Lyer-consistent line segments should have an illusion as great as or greater than the standard version (which contains both consistent and inconsistent line segments). The tilt constancy theory would make the opposite prediction: The standard Ponzo figure should have a greater illusion than the Müller-Lyer-consistent version. The reason for this prediction is that in the standard version, there are clear contours on the left and right to locally perturb orientation perception. In the Müller-Lyerconsistent version, these contours have been distorted. Hence, we were able to create a situation in which the two theories made opposite predictions.

\section{Method}

The procedure was identical to that in the previous experiments, except for the following. The observers were tested for 12 trials with the standard Ponzo version, and for 12 trials with the MüllerLyer version. The order of trials was randomly determined for each observer. The same 16 observers participated in this experiment as in Experiment 2B. Half participated in Experiment 3 first, and half in Experiment 2B first. The dimensions of the standard Ponzo were identical to those in the previous experiments, and the stimuli in Figure 9 are drawn to scale.

\section{Results and Discussion}

The normal Ponzo figure exhibited a significantly larger illusion than did the Müller-Lyer version $(9.34 \%$ vs. $6.63 \%$ illusion, respectively). This difference was reliable $[t(15)=4.55, p<.01]$. The variability of setting was similar with the two figures. The standard deviation of the 12 settings, averaged over the 16 observers, was $4.0 \%$ and $3.3 \%$ for the standard and the Müller-Lyer versions of the Ponzo, respectively.

In this experiment, the assimilation theory and the tilt constancy theory made different predictions. The assim- ilation theory claims that the Ponzo illusion is caused by the same mechanism as the Müller-Lyer illusion. Hence, a version of the Ponzo illusion that is more similar to the Müller-Lyer illusion should exhibit a greater illusion. The tilt constancy theory made the opposite prediction. The normal version has consistent oblique contours with which to affect orientation perception, whereas in the MüllerLyer version, the contours are not as clear. The results favored the tilt constancy theory over the assimilation theory.

One might object that the Müller-Lyer version (Figure 9B) is not a fair test of Pressey's theory, because the Müller-Lyer illusion itself would be reduced if there were a gap between the wings out and the shaft. Note, however, that the Ponzo is normally drawn with a gap between the top line and the context lines. In this regard, it is interesting to note that the "Ponzo" stimuli used by Pressey and his colleagues do not contain a gap between the context lines and the top horizontal line (e.g., Pressey et al., 1971; Pressey \& Epp, 1992). Thus, both our standard and our Müller-Lyer versions are more similar to the Ponzo illusion, as it is normally depicted, than is that used by Pressey and his colleagues. It may be possible to draw a version of the Ponzo figure that is more similar to the Müller-Lyer illusion. For the typical Ponzo figure, however, making it similar to the Müller-Lyer figure does not increase the illusion; rather, it decreases it.

Of course, the Müller-Lyer illusion, whatever its cause, is an extremely robust illusion. However, Experiment 3 demonstrated that an explanation of the Ponzo illusion in terms of the mechanisms that cause the Müller-Lyer illusion is neither necessary nor sufficient. We frankly have no idea of the cause of the Müller-Lyer illusion. That illusion may be due to some form of assimilation, as was suggested by Pressey and his colleagues. Alternatively, it may be caused by the inappropriate application of a sizeconstancy mechanism (Gregory \& Harris, 1975). Whatever its cause, we believe that it is not related to the Ponzo illusion, for three reasons. First, in a factor-analytic study, Coren, Girgus, Erlichman, and Hakstian (1976) found that the Müller-Lyer illusion and the Ponzo illusion were not classified together: Observers who show a large Ponzo illusion are not the same observers as those who show a large Müller-Lyer illusion (cf. our findings in Experiment 1). Second, although both the Müller-Lyer illusion (Coren \& Girgus, 1978, p. 31) and the Ponzo illusion (Fisher, 1968b, 1973) vary in streng th as a function of the angle between the components, there are fundamental differences. The Müller-Lyer illusion generally increases monotonically as the angle becomes more acute (as long as the shaft lines are not too long), whereas the strength of the Ponzo illusion first increases, then decreases with the angle. Finally, Coren (1986) has shown that it is not the angle between the shaft and the arrowheads that is critical, but how far from the shaft the arrowheads extend. Longer arrowheads and more acute angles are equivalent in their effect on the Müller-Lyer illusion. In fact, it has been long known that any blobs at the ends of the shaft can cause a Müller-Lyer-like illusion. Thus, 
there are ample empirical grounds for believing that the Ponzo and the Müller-Lyer illusions are caused by different mechanisms.

In retrospect, Experiment 3 could also be considered a test comparing our tilt constancy theory with the lowpass filter theory and the pool-and-store theory. In the present experiment, we emphasized the assimilation theory. The assimilation theory predicted a larger illusion in the Müller-Lyer version (Figure 9B), whereas we predicted a larger illusion in the standard version (Figure 9A). Low-pass filtering is one possible mechanism to account for assimilation. Indeed, depending on the parameters of the model, low-pass filtering might predict a larger illusion in the Müller-Lyer version (Figure 9B) than in the standard version (Figure 9A), because in a filtered representation, the center of mass of the blob formed by the apex line and the context lines would be farther from the center of the figure. The results were consistent with the tilt constancy theory and inconsistent with the lowfrequency, pool-and-store, and assimilation theories.

\section{EXPERIMENT 4}

Probably the most popular and influential theories of the Ponzo illusion make reference to depth perception, linear perspective, and/or size constancy. There are actually four or five related theories (Green \& Hoyle, 1963), but we will mention only two. According to one version, a Ponzo stimulus triggers a linear perspective mechanism so that the apex of the figure is perceived as being farther from the observer than is the base of the figure. Inappropriate application of size constancy therefore makes the apex line (usually a horizontal line on the top) appear longer (Gregory, 1963, 1968). ${ }^{3}$ Alternatively, the application of the linear perspective mechanism, without perceived depth, could be responsible for the illusion (Gillam, 1980). According to this view, the foreshortening caused by linear perspective causes the illusion (Gillam, 1973). For the purposes of Experiment 4, all of the theories that make reference to depth perception and/or linear perspective make the same prediction, so we will simply call them the perspective family of theories.

The predictions of the perspective family can be seen in reference to Figure 10, Condition 1 . The figure includes the Ponzo figure (vertical lines on the left), and it also could be interpreted in terms of linear perspective. One could imagine looking down a long hallway, with the small rectangle in the center as the distant end of the hallway. The right vertical line could be interpreted as being farther from the observer than the left vertical line. Since the two vertical lines subtend the same visual angle in the figure, the apex line (right vertical line) should appear longer, according to the perspective family of theories. Most observers will perceive the right line to be longer than the left line, consistent with the Ponzo illusion.

The tilt constancy theory makes the same prediction for Figure 10, Condition 1. When judging the height of the tops of the lines, local context (the oblique line) will
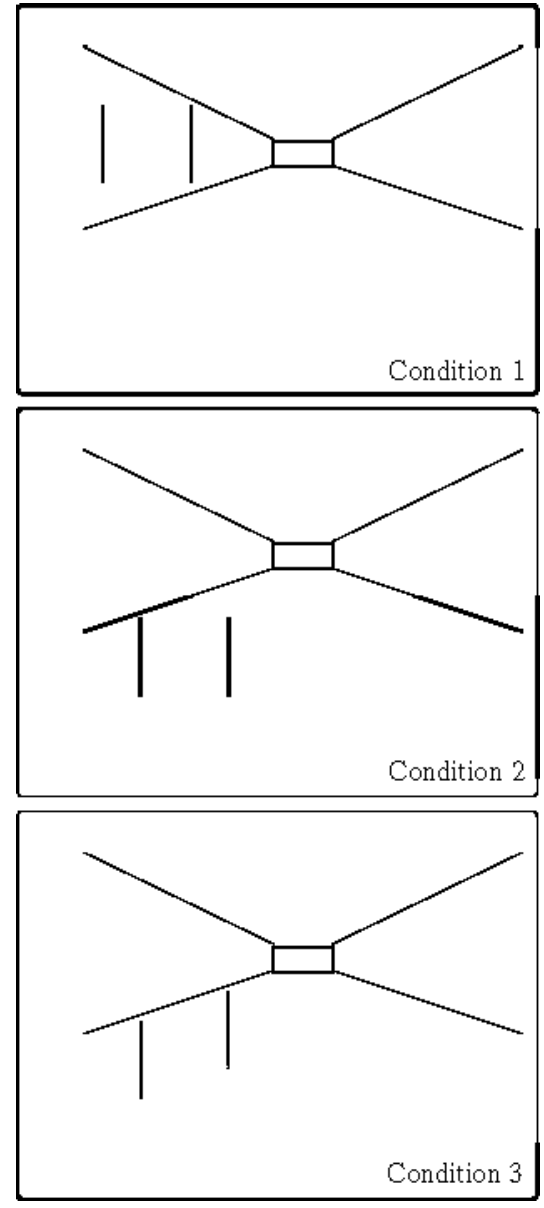

Figure 10. The stimuli used in Experiment 4.

make the top of the right line appear higher. When judging the bottoms of the lines, local context (the oblique line) will make the bottom of the right line appear lower. Since both theories make the same prediction for Condition 1 (and the Ponzo illusion in general), we created stimuli for which each theory would make a unique prediction.

In judging the length of the two vertical lines in Condition 2 (Figure 10) the tilt constancy theory reverses its prediction. Because of local context, the top of the left line will be perceived as higher, and therefore the left line will be perceived as longer. Furthermore, the strength of the effect should be about half of that for Condition 1, because there is only one oblique line to affect the observer's perception. It is unclear what the perspective theories would predict. Perhaps the simplest prediction is that since the two lines are at equal height in the picture, they are at the same distance from the observer and, therefore, should be perceived as equal in length.

The predictions of the perspective family of theories are clear in Condition 3, however. The line on the right should be perceived as farther from the observer, because of linear perspective and the adjacency principle (Gogel, 
1978). The adjacency principle states that the perceived distance of an object at an unknown distance will be the same as that of objects near it. Hence, the line on the right should be perceived as longer (see Figure 10, Condition 3 ). Furthermore, the magnitude of the illusion should be the same as that in Condition 1, because the distance between the lines is identical to that in Condition 1 . The tilt constancy theory predicts no difference between the perceived lengths of the lines, since the effect of local context will be identical.

\section{Method}

Each observer was run for a single block of 24 trials. There were 8 trials of each condition randomly intermixed within the block. The task was to adjust the length of the vertical line on the left to match the length of the line on the right, using the arrow keys on the keyboard, as in the previous experiments. Note, however, that unlike the previous experiments, the base line (left vertical line) was fixed in length, and the observer's task was to adjust the length of the line on the right. Also, in each condition, the top of the adjustable line was fixed, and the bottom of the line on the right became longer or shorter as the observer pressed the arrow keys on the keyboard. ${ }^{4}$

The stimulus patterns were placed on the monitor in the position shown in Figure 10. The figure is accurately drawn to scale; the outline of the panels represents the edge of the viewable area of the monitor. The standard line (vertical line on the left) subtended a visual angle of approximately $4.6^{\circ}$ (120 pixels). The length of the adjustable line was randomly set at the beginning of each trial, as before. Twenty-four observers participated. In all other respects, the experiment was identical to the previous experiments.

\section{Results}

In Condition 1, in which the lines are objectively the same length, both theories predict that observers will perceive the left line as longer. Therefore, observers should adjust the line too short. Indeed, each of $24 \mathrm{ob}-$ servers adjusted the right line too short. The mean magnitude of the illusion was $-4.94 \%$ (the sign indicating that the line was adjusted too short).

In Condition 2, the orientation theory predicted that observers should make the opposite error; they should adjust the line too long. Twenty of 24 observers adjusted the line too long. The mean illusion error was $+2.38 \%$, as would be predicted by the tilt constancy theory; this illusion was about half of the illusion in Condition 1 , but it was significantly greater than zero $[t(23)=4.96, p<.05]$.

Finally, the linear perspective family of theories predicted the same illusion in Condition 3 as in Condition 1; the observers should have adjusted the line too short. Only 8 of 24 observers adjusted the line too short. The average magnitude of the illusion was $+0.881 \%$, not significantly different from zero $[t(23)=1.49]$.

By analysis of variance, the illusion magnitude significantly differed by condition $[F(2,46)=71.34, p<.01]$. The standard deviation of the eight settings (per condition), averaged over the 24 observers, was $1.9 \%, 1.9 \%$, and $3.6 \%$ for Conditions $1-3$, respectively. In summary, both theories predicted the results in Condition 1 . The prediction of the tilt constancy theory for Condition 2 was

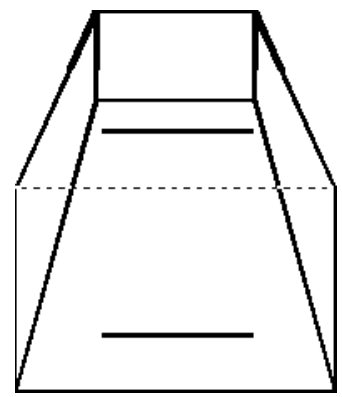

Figure 11. When viewed as a truncated pyramid from above, the top line still appears longer than the bottom line.

supported, whereas the prediction of the linear perspective family of theories for Condition 3 was not supported.

\section{Discussion}

Experiment 4 demonstrates that an explanationin terms of linear perspective and size constancy is neither necessary nor sufficient to account for the Ponzo illusion. Of course, we are not arguing that the mechanisms responsible for the processing of linear perspective and/or size constancy do not play a role in perception. Indeed, perceptual errors induced by the misapplication of size constancy can be powerful. Furthermore, these other mechanisms may, under special circumstances, affect or contribute to the perception of Ponzo-like stimuli. For example, elaborating a Ponzo stimulus so that it appears three-dimensional (with, e.g., additional perspective cues) can enhance the illusion. However, as Newman and Newman (1974) correctly point out, increasing the size of the illusion by adding a factor does not logically mean that that factor is the cause of the illusion; it simply adds another effect.

The fact that another factor, such as perceived depth, may affect the Ponzo illusion without causing the basic illusion is illustrated in Figure 11 (see Rock, 1984, p. 156). If Figure 11 is perceived as a pyramid from above, the upper line appears longer, even though it is perceived as being closer than the lower line. Thus, there is some factor operating in opposition to perceived depth. We believe that factor involves local orientation cues. Of course, Figure 11 is ambiguous, and the lower line may be perceived as closer than the top line. Perceived depth, operating with orientation, can increase the effect. The fact that perceived depth does not reverse the effect when the figure is perceived as a pyramid demonstrates that it is not a sufficient explanation for the Ponzo illusion.

Figure 11 also illustrates an interesting qualification to the linear perspective family of theories. None of the observers in Experiment 4 spontaneously remarked that the stimuli (Figure 10) looked three-dimensional (they were not asked). However, several visual scientists, upon seeing the stimuli in Figure 10, commented that they are ambiguous (like Figure 11). They may be perceived as if looking down a long hallway or as a pyramid viewed from 
above. The illusion in Figure 10 (Condition 1) remains, regardless of how the figure is interpreted. If the depth relations in Figure 10 are ambiguous, the depth relations in the basic Ponzo illusion must also be ambiguous, and the linear perspective family of theories should specify why one interpretation is favored.

The above discussion highlights a fundamental difference between the orientation and the perspective approaches to the Ponzo illusion. For the tilt constancy theory, the most important stimulus component is the oblique contour nearest the stimulus. A single oblique line can affect orientation perception (e.g., Andriessen \& Bouma, 1976) and thus can explain why a Ponzo illusion can occur with just a single oblique line (Fisher, 1968a). Linear perspective, on the other hand, requires at least two lines. In terms of perspective, a single oblique line is ambiguous. We could have performed Experiment 4 with less elaborate stimuli, but a minimum of two context lines would be required to represent perspective. Thus, the linear perspective theory cannot explain a Ponzo illusion created with a single line (Fisher, 1968a).

There are, of course, other problems with the sizeconstancy account of the Ponzo illusion. If the test lines are rotated $90^{\circ}$, the illusion disappears (e.g., Gillam, 1980). That is, for example, if the vertical bars in Figure 1 are replaced by horizontal bars, there is no Ponzo illusion. These problems led Gillam (1973) to propose that it is not size scaling that causes the Ponzo illusion, but foreshortening scaling. However, foreshortening scaling makes the same predictions as size scaling for Experiment 4 and, therefore, is also inconsistent with the results of Experiment 4.

\section{GENERAL DISCUSSION}

Of the six theories of the Ponzo illusion tested in this investigation, the tilt constancy theory provided the best account of the findings. Experiment 1 demonstrated that the misperception of vertical alignment caused an illusion of the same magnitude as the Ponzo illusion. Furthermore, performance on the tilt induction task was highly correlated with the magnitude of the Ponzo illusion. Experiments $2 \mathrm{~A}$ and $2 \mathrm{~B}$ compared predictions of the tilt constancy theory with the low spatial frequency theory, a size-comparison theory, and the pool-and-store model. For these alternative theories, the orientation of the context lines is not important. The only important stimulus feature is the size of the gap between the context lines and the test lines. In this experiment, however, the presentation of oblique context lines-that is, those that distort orientation - was necessary to produce a Ponzo illusion. In Experiment 3, we tested predictions of the assimilation model by comparing the standard Ponzo figure with one consistent with the Müller-Lyer illusion. A standard Ponzo figure with oblique context lines produced a larger illusion than did a stimulus that was consistent with the Müller-Lyer illusion. In the final experiment, we compared predictions of the tilt constancy

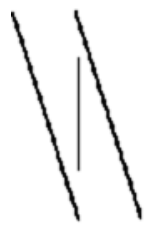

A

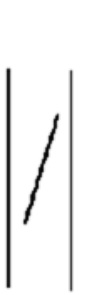

B
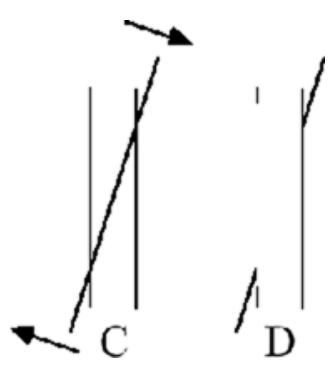

$\mathrm{D}$
Figure 12. The figure demonstrates the relation between the tilt induction effect and the Poggendorff illusion. In the tilt induction effect, (A) a vertical line appears slanted in the direction opposite the context lines (i.e., clockwise); (B) a slanted line appears to be tilted to a greater extent than it actually is; $(C)$ the arrows indicate the direction of the misperception of orientation in panel B ; (D) the Poggendorff illusion results in each of the oblique line segments' appearing to be tilted to a greater extent than they are, causing them to appear misaligned.

theory with those of the linear perspective family of theories. In this experiment, the stimuli were drawn in accordance with the rules of linear perspective. The only theory that was consistent with the entire set of experiments was the tilt constancy theory. Undoubtedly, there are theories that we have not explicitly mentioned. However, the present results provide constraints for all existing and future theories of the Ponzo illusion.

There are a number of other illusions that can be explained in a similar manner. The relation between the tilt induction effect and the Poggendorff illusion can be seen in Figure 12.5 Figure 12A depicts the tilt induction effect in which a vertical line, surrounded by oblique lines, is perceived as tilted in a clockwise direction. Gibson (1937) also reported the same illusion for an oblique line surrounded by vertical lines: The oblique line was perceived as having a greater tilt than it actually had (Figure 12B). The direction of the illusion is indicated with arrows in Figure 12C. Finally, in Figure 12D, the center portion of the oblique line has been occluded. If the center portion of the oblique line is occluded and the orientation of the visible parts is misperceived, the result is the Poggendorff illusion - the oblique line segments will not be perceived as collinear.

In several ways, the Poggendorff illusion behaves similarly to the tilt induction, Zöllner, and Ponzo illusions. Like these other illusions, its magnitude is greater when it is presented in a vertical rather than horizontal orientation (e.g., Leibowitz \& Toffey, 1966), and it is also greater when the angle between the context lines and the test lines are in the same range as the Zöllner illusion (e.g., Green \& Hoyle, 1964; Greene \& Pavlow, 1989; Velinsky, 1925).

Many other illusions appear to be related to the Zöllner figure (see Fisher, 1968b, for other examples that might fit this class of illusion). The bending of the horizontal lines in the Wündt-Hering illusion (Figure 1) illustrates two properties of the tilt induction effect (and the Zöllner illusion). First, the degree of distortion is re- 

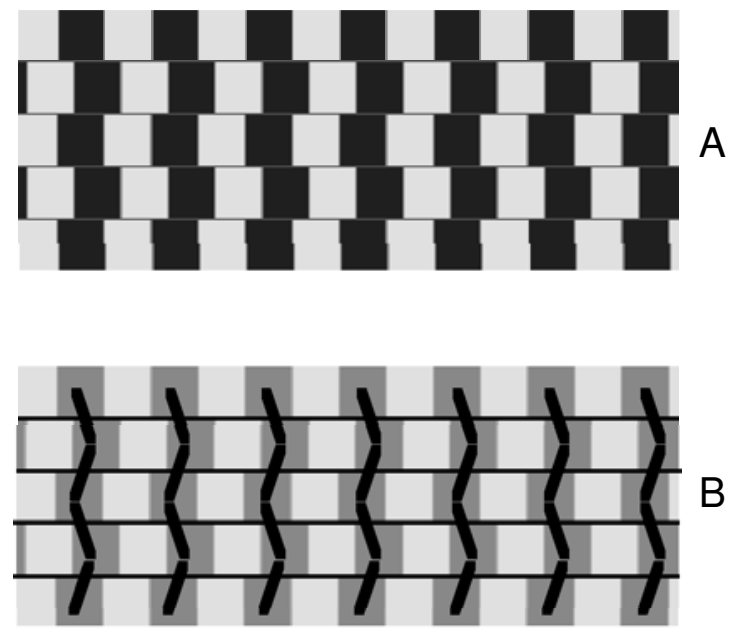

Figure 13. The cafe wall illusion could be considered an example of the Zöllner illusion. (A) In the cafe wall illusion, the mortar lines between the bricks do not appear to be straight. (B) The pattern of the dark bricks may have the same effect as the oblique lines in the Zöllner illusion.

lated to the angle between the context lines and the horizontal lines, yielding the perception of curvature. Note that linear perspective makes the wrong prediction for the Wündt-Hering illusion. If the point at which the oblique lines meet is the vanishing point, horizontal lines should appear horizontal. Second, the effects are local, so that the extent of bend is mostly determined by the closest context line. Speculatively, we would like to add the possibility that the cafe wall illusion could be considered an example of the Zöllner illusion. This possibility is illustrated in Figure 13, in which the dark bricks may provide the oblique lines. One could also include in the group of illusions related to the Zöllner illusion the Fraser spiral. The Fraser twisted cord, however, probably has a different origin (see Tyler \& Nakayama, 1984).

It should be noted that our account of the Zöllner and Poggendorff illusions is not a theory of the misperception of angles but, rather, a theory of the misperception of orientation. By angles, we mean a stimulus that contains actual lines that meet at a point or nearly meet at a point. In 1861, Hering proposed that acute angles are always overestimated (Hering, 1861). Although this theory cannot account for the Ponzo illusion, it has been used to explain the Zöllner, Poggendorff, and related illusions. In modern physiological terms, similar orientation-tuned neural units mutually inhibit each other, resulting in a larger perceived angle (Blakemore, Carpenter, \& Georgeson, 1970). This theory cannot be correct for the Zöllner or Poggendorff illusions, because both of these illusions can be obtained without intersecting lines or even lines that come close to intersecting (e.g., Pressey \& Sweeney, 1972; Tyler \& Nakayama, 1984; Wilson \& Pressey, 1976). We hypothesize that these illusions are due to a misper- ception of orientation, and thus our explanation does not require that the stimulus contain explicit angles.

The tilt constancy theory claims that these illusions are due to the misperception of orientation owing to visual context. The effect of visual context on the perception of vertical and horizontal is demonstrated by the tilt induction effect. The relevance of visual context to the sense of orientation in a naturalistic setting was dramatically illustrated by Asch and Witkin (1948). Observers looked into a room that was rotated $22^{\circ}$. Observers' perception was influenced by the room so that the perception of vertical and horizontal was distorted in the direction of the tilted environment. This illusion, called the tilted room illusion, can be experienced at several roadside attractions and amusement parks (Banta, 1995; Knight, 1999; Shimamura \& Prinzmetal, 2000; see also www. illusionworks.com). Howard has recently demonstrated that that the visual context can override shifts in gravity relative to body position (Howard, 1998; Howard \& Childerson, 1994). The tilted room illusion may be related to the pitch-box effect (Kleinhans, 1970; Matin \& Li, 1995; Stoper \& Cohen, 1986).

Witkin and Asch (1948) obtained a similar effect with a simple luminous rod and frame (i.e., the rod-and-frame effect). Coren and Hoy (1986) showed that a rod-andframe effect could be obtained with stimuli nearly identical to those used by Gibson (1937). Note that this account of visual illusions refers to a normally adaptive process. Visual context normally provides veridical and stable information about orientations in the environment.

We have suggested that the mechanisms responsible for the tilted room illusion are the same as those responsible for other illusions of orientation (including the Ponzo illusion). This claim is controversial. On the one hand, Day (1972) suggested that the tilt induction effect, the Zöllner illusion, and the tilted room illusion of Asch and Witkin (1948) were all caused by the same mechanism. On the other hand, Howard proposed that although the Zöllner illusion and the tilt induction effect were caused by one mechanism, context illusions that involved large stimuli (the rod-and-frame effect and the tilted room illusion) were caused by a different mechanism. Four of the reasons given by Howard for believing that the large and the small context illusions are caused by different mechanisms are discussed below (Howard, 1982, pp. 155156). After considering these reasons, we have come to the conclusion that either the evidence supports Day's claim that the same mechanisms are involved or the evidence is equivocal.

First, Howard (1982) asserts that the large-scale illusions (tilted room, rod-and-frame effect) give much larger effects than the small-scale illusions (e.g., Zöllner illusion, tilt induction effect). He cites the finding of Asch and Witkin (1948) of a $20^{\circ}$ error in setting a rod to vertical (or horizontal) in the tilted-room illusion. The tilt induction effect is much smaller. However, the $20^{\circ}$ error cited by Howard was found in very particular circum- 
stances (see Asch \& Witkin, 1948). For example, the observer was seated in a chair that was tilted $24^{\circ}$. The observers went through an elaborate 7 -min indoctrination period so that they would accept the room as upright. Under less unusual circumstances, Asch and Witkin reported a much smaller illusion. Indeed, Wenderoth (1974) has shown that the magnitude of the rod-and-frame effect is often overestimated. When estimated correctly, it is similar to the tilt induction effect. We have measured the tilted room illusion in a naturalistic context and found that it can be similar in magnitude to the tilt induction effect in the laboratory (Shimamura \& Prinzmetal, 2000). For the tilt induction effect, the larger the context, the larger the illusion (Johnstone \& Wenderoth, 1989; Shimamura \& Prinzmetal, 1999; Wenderoth \& Johnstone, 1988). The only generalization that emerges is that the greater the amount of context a stimulus contains, the larger the orientation illusion.

The second reason that Howard gives for believing that the large and the small context effects are different is that the large context effects show a special role of peripheral presentation. The evidence for this assertion is a comprehensive series of experiments by Ebenholtz and his colleagues, demonstrating that the magnitude of the rod-and-frame effect increases as the stimulus gets retinally larger (e.g., Ebenholtz, 1977, 1985; Ebenholtz \& Callan, 1980). As the stimulus gets larger, the frame falls more in the periphery. In contrast, Muir and Over (1970) found that the tilt-after effect did not vary with eccentricity. However, this comparison is flawed. Muir and Over used the same stimulus presented at different eccentricities, whereas with the rod-and-frame task, increasing the size of the frame increase its eccentricity and its size. As was mentioned above, increasing the size of the tilt induction stimulus increases the magnitude of the illusion.

Third, Howard argues that the tilt induction effect (and the Zöllner illusion) is greatly diminished when the context is separated from the test line (e.g., Johnstone \& Wenderoth, 1989), whereas with the rod-and-frame task, context (the frame) can have an influence over large distances. Note, however, that the rod-and-frame task behaves in exactly the same manner as the tilt induction effect: As the gap between the rod and the frame increases, the effect diminishes (Coren \& Hoy, 1986; Zoccolotti, Antonucci, \& Spinelli, 1993). Clearly, quantitative comparisons about the magnitude of the effect of distance are difficult to make, because a small gap between the context and the test line in the fovea may have a larger effect than a large gap in the periphery. Note that increasing the distance between the context and the test portions of the stimulus reduces the magnitude of both the Zöllner (Oyama, 1975; Wallace, 1969) and the Ponzo (Fisher, 1968b) illusions.

Finally, Howard argues that the tilted-room illusion involves cognitive factors, such as the degree of realism in the tilted scene. However, he points out that the tilt induction effect does not involve familiar objects. (Note that, by this argument, the rod-and-frame effect would be caused by a different mechanism than the tilted room illusion.) Howard has persuasively argued elsewhere that cognitive variables come into play when the orientation of the stimulus becomes ambiguous (Howard \& Childerson, 1994). Thus, when the rotation of a room, frame, or grating approaches $45^{\circ}$, directions (e.g., top, bottom, left, right) become ambiguous. Cognitive factors, such as the intrinsic orientation of real-world objects, may disambiguate orientation. We simply do not know whether cognitive factors would similarly influence the tilt induction effect as the stimulus orientation approaches $45^{\circ}$. This hypothesis has not been tested.

Recently, Prinzmetal and Beck (in press) have tested the notion that the Ponzo, Zöllner, Poggendorff, and tilt induction illusions are caused by the same mechanism as the tilted room illusion and the rod-and-frame effect. They reasoned that if the same mechanisms are responsible for the small-scale illusions as for the tilted room illusion, variables that affect the tilted room illusion should similarly affect these other illusions. Asch and Witkin discovered that tilting the observer increased both the tilted room illusion and the rod-and-frame effect (Asch \& Witkin, 1948; Witkin \& Asch, 1948; see also DiLorenzo \& Rock, 1982). Presumably, tilting observers makes them rely more on visual cues to orientation and less on gravity-based cues. Prinzmetal and Beck discovered that tilting observers increased the Ponzo, Zöllner, Poggendorff, and tilt induction illusions but did not affect a control condition (the Müller-Lyer illusion). Thus, a critical variable that influences the tilted room illusion affects other orientation illusions, suggesting that a common mechanism may underlie both types of phenomena. Other accounts of the Ponzo illusion would not predict this result.

Note that, like theorists before us, we attribute these orientationillusions to normally adaptive processes. Normally, visual cues to orientations in the world help us maintain a stable representation of orientation, despite changes in retinal orientation. It is only in the tilted roomas demonstrated in the antigravity house-that these normally adaptive cues to orientation lead us astray. These same processes can use local information to determine orientation, and it is the local cues to orientation that cause the Ponzo illusion.

\section{REFERENCES}

Andriessen, J. J., \& Bouma, H. (1976). Eccentric vision: Adverse interactions between line segments. Vision Research, 16, 71-78.

Asch, S. E., \& Witkin, H. A. (1948). Studies in space orientation: II. Perception of the upright with displaced visual fields and with body tilted. Journal of Experimental Psychology, 38, 455-475.

BANTA, C. (1995). Seeing is believing: Haunted shacks, mystery spots, and other delightfulphenomena. Agoura Hills, CA: Funhouse Press.

Blakemore, C., Carpenter, R. H., \& Georgeson, M. A. (1970). Lateral inhibition between orientation detectors in the human visual system. Nature, 228, 37-39.

Boring, E. G. (1942). Sensation and perception in the history of experimental psychology. New York: Appleton-Century-Crofts.

Carlson, C. R. Moeller, J. R, \& Anderson, C. H. (1984). Visual illusions without low spatial frequencies. Vision Research, 24, 1407 1413. 
Coren, S. (1986). An efferent component in the visual perception of direction and extent. Psychological Review, 93, 391-410.

Coren, S., \& Girgus, J. S. (1978). Seeing is deceiving: The psychology of visual illusions. Hillsdale: NJ: Erlbaum.

Coren, S., Girgus, J. S., Erlichman, H., \& Hakstian, A. R. (1976). An empirical taxonomy of visual illusions. Perception \& Psychophysics, 20, 129-137.

Coren, S., \& Hoy, V. S. (1986). An orientation illusion analog to the rod and frame: Relational effects in the magnitude of the distortion. Perception \& Psychophysics, 39, 159-163.

DAY, R. H. (1972). Visual spatial illusions: A general explanation. Science, 175, 1335-1340.

DeValois, R. L., \& DeValois, K. K. (1990). Spatial Vision. New York: Oxford University Press.

DiLorenzo, J., \& Rock, I. (1982). The rod-and-frame effect as a function of the righting of the frame. Journal of Experimental Psychology: Human Perception \& Performance, 8, 536-546.

Ebenholtz, S. M. (1977). Determinants of the rod-and-frame effect: The role of retinal size. Perception \& Psychophysics, 22, 531-538.

Ebenholtz, S. M. (1985). Absence of relational determination in the rod-and-frame effect. Perception \& Psychophysics, 37, 303-306.

Ebenholtz, S. M., \& Callan, J. W. (1980). Modulation of the rodand-frame effect: Retinal size vs. apparent size. Psychological Research, 42, 327-334

FisHER, G. H. (1968a). An experimental comparison of rectilinear and curvilinear illusions. British Journal of Psychology, 59, 23-28.

Fisher, G. H. (1968b). Gradients of distortion seen in the context of the Ponzo illusion and other contours. Quarterly Journal of Experimental Psychology, 13, 212-217.

FisHeR, G. H. (1969). Towards a new explanation of contours which induce illusory distortion. British Journal of Psychology, 60, 179-185.

FisHeR, G. H. (1973). Toward a new explanation for the geometrical illusions: II. Apparent depth or contour proximity. British Journal of Psychology, 64, 607-621.

GiBson, J. J. (1937). Adaptation, after-effect, and contrast in the perception of tilted lines: II. Simultaneous contrast and the areal restriction of the after-effect. Journal of Experimental Psychology, 20, 553-569.

GibSON, J. J., \& RADNER, M. (1937). Adaptation, after-effect and contrast in the perception of tilted lines: I. Quantitative studies. Journal of Experimental Psychology, 20, 453-467.

Gillam, B. (1973). The nature of size scaling in the Ponzo and related illusions. Perception \& Psychophysics, 14, 353-357.

Gillam, B. (1980, January). Geometrical illusions. Scientific American, 242, 102-111.

GinsBURG, A. P. (1984). Visual form perception based on biological filtering. In L. Spillmann \& B. R. Wotten (Eds.), Visual form perception based on biological filtering (pp. 53-72). Hillsdale, NJ: Erlbaum.

Girgus, J. S., \& Coren, S. (1982). Assimilation and contrast illusions: Differences in plasticity. Perception \& Psychophysics, 32, 555-561.

GogeL, W. C. (1978, May). The adjacency principle in visual perception. Scientific American, 238, 126-139.

Gogel, W. C., \& Sturm, R. D. (1972). A test of the relational hypothesis of perceived size. American Journal of Psychology, 85, 201-216.

Green, R. T., \& Hoyle, E. M. (1963). The Poggendorff illusion as a constancy phenomenon. Nature, 200, 611-612.

Green, R. T., \& Hoy Le, E. M. (1964). The influence of spatial orientation on the Poggendorff illusion. Acta Psychologica, 22, 348-366.

Greene, E., \& Pavlow, G. (1989). Angular induction as a function of contact and target orientation. Perception, 18, 143-154.

GREGORY, R. L. (1963). Distortion of visual space as inappropriate constancy scaling. Nature, 199, 678-680.

Gregory, R. L. (1968, November). Visual illusions. Scientific American, 219, 66-76.

Gregory, R. L., \& HARRIS, J. P. (1975). Illusion-destruction by appropriate scaling. Perception, 4, 203-220.

Hering, E. (1861). Beitrage zur psychologie [Contributions to psychology]. Leipzig: Engleman.

Howard, I. P. (1982). Human visual orientation. New York: Wiley.

HowARD, I. P. (1998, December). Knowing which way is up. Presentation at the Vision Science Symposium, University of California, Berkeley, December 15, 1998.
Howard, I. P., \& Childerson, L. (1994). The contribution of motion, the visual frame, and visual polarity to sensations of body tilt. Perception, 23, 753-762.

Johnstone, S., \& Wenderoth, P. (1989). Spatial and orientation specific integration in the tilt illusion. Perception, 18, 5-23.

Jordan, K., \& RANDALL, J. (1987). The effects of framing ratio and oblique length in the Ponzo illusion magnitude. Perception \& Psychophysics, 41, 435-439.

KLeinhans, J. L. (1970). Perception of spatial orientation in sloped, slanted, and tilted visual field. Unpublished doctoral dissertation, Rutgers University, New Brunswick, NJ.

KNight, J. (1999). The world turned upside down. New Scientist, 161, 34-42.

KRAMER, K. S. (1978). Inhibitory interactions as an explanation of visual orientation illusions. Unpublished doctoral dissertation, UCLA.

KÜNNAPAS, T. M. (1955). Influence of frame size on apparent length of a line. Journal of Experimental Psychology, 50, 168-170.

Leibowitz, H., \& Toffey, S. (1966). The effect of rotation and tilt on the magnitude of the Poggendorfillusion. Vision Research, 6, 101-103.

Matin, L., \& LI, W. (1995). Multimodal basis for egocentric spatial localization and orientation. Journal of Vestibular Research, 5, 499-518.

Muir, D., \& Over, R. (1970). Tilt aftereffect in central and peripheral vision. Journal of Experimental Psychology, 85, 165-170.

Newman, C. V., \& Newman, B. M. (1974). The Ponzo illusion in pictures with and without suggested depth. American Journal of Psychology, 87, 511-516.

Oyama, T. (1975). Determinants of the Zöllner illusion. Psychological Research, 37, 261-280.

Post, R. B., Welch, R. B., \& Caufield, K. (1998). Relative spatial expansion and contraction within the Müller-Lyer and Judd illusions. Perception, 27, 827-838.

Pressey, A. W., Butchard, N., \& Scrivner, L. (1971). Assimilation theory and the Ponzo illusion: Quantitative predictions. Canadian Journal of Psychology, 25, 486-497.

Pressey, A. W., \& EPP, D. (1992). Spatial attention in Ponzo-like patterns. Perception \& Psychophysics, 52, 211-221.

Pressey, A. W., \& Sweeney, O. (1972). Acute angles and the Poggendorff illusion. Quarterly Journal of Experimental Psychology, 24, 169-174.

Prinzmetal, W., \& Beck, D. M. (in press). Tilt-constancy theory of visual illusions. Journal of Experimental Psychology: Human Perception \& Performance.

Prinzmetal, W., \& Gettleman, L. (1993). Vertical-horizontal illusion: One eye is better than two. Perception \& Psychophysics, 53, 81-88.

Rock, I. (1984). Perception. New York: Scientific American Library.

Rock, I. (1995). Perception. New York: Scientific American Library.

Rock, I., \& Ebenholtz, S. (1959). The relational determination of perceived size. Psychological Review, 66, 387-401.

Shimamura, A. P., \& Prinzmetal, W. (1999). The mystery spot illusion and its relation to other visual illusions. Psychological Science, 10, 501-507.

Stoper, A. E., \& Cohen, M. M. (1986). Judgments of eye level in light and in darkness. Perception \& Psychophysics, 40, 311-316.

Tyler, C. W., \& Nakayama, K. (1984). Size interactions in the perception of orientation. In L. Spillman \& B. R. Wooten (Eds.), Sensory experience, adaptation, and perception: Festschrift for Ivo Kohler (pp. 529-546). Hillsdale, NJ: Erlbaum.

VELINSKY, S. (1925). Explication physiologique de l'illusion de Poggendorff [Physiological explanation of the Poggendorff illusion]. Année Psychologique, 26, 107-116.

WALLACE, G. K. (1969). The critical distance of interaction in the Zöllner illusion. Perception \& Psychophysics, 5, 261-264.

Wallace, G. K., \& Moulden, B. (1973). The effect of body tilt on the Zöllner illusion. Quarterly Journal of Experimental Psychology, 25, $10-21$.

Wenderoth, P. M. (1974). The distinction between the rod-and-frame illusion and the rod-and-frame test. Perception, 3, 205-212.

Wenderoth, P. \{M.\}, \& Johnstone, S. (1988). The mechanism of the direct and indirect tilt illusions. Vision Research, 28, 301-312.

Wilson, A. E., \& Pressey, A. W. (1976). The role of apparent distance in the Poggendorffillusion. Perception \& Psychophysics, 20, 309-316. 
Witkin, H. A., \& Asch, S. E. (1948). Studies in space orientation: IV. Further experiments of the upright with displaced visual fields. Journal of Experimental Psychology, 38, 762-782.

Zoccolotti, P., Antonucci, G., \& SPinelli, D. (1993). The gap between rod and frame influences the rod-and-frame effect with small and large inducing displays. Perception \& Psychophysics, 54, 14-19.

\section{NOTES}

1. In order to obtain the finest resolution, each keyboard response caused the line to grow or shrink by 1 pixel. Hence, for the Ponzo task, on every other response, the base line was 1 pixel off-center.

2. Because the shape of the gap between the context and the horizontal lines is different for the two figures, it was difficult to precisely equate the two. To be conservative, we made the gap at the top smaller in the rectilinear version than in the standard version. Thus, there would be more blurring into the context in the rectilinear version. In the stan- dard version, there were 30 white pixels in the gap on each end; in the rectilinear version, there were 24 pixels.

3 . Gregory's theory does not necessarily require the conscious perception of depth (personal communication, July, 1999). Experiment 4 addresses all theories that make reference to linear perspective, regardless of whether or not they make reference to the explicit perception of depth triggered by linear perspective.

4. We also ran an experiment in which the top and bottom of the adjustable line moved, as in the previous experiments. The results were identical.

5. Historically, the Zöllner and Poggendorff illusions are related. In 1860, Zöllner submitted his illusion for publication. The journal editor, Poggendorff, noticed that the oblique lines did not appear to be collinear (Boring, 1942, p. 260).

(Manuscript received August 31, 1999; revision accepted for publication February 25, 2000.) 